\title{
A comparison of commercially available demineralized bone matrices with and without human mesenchymal stem cells in a rodent spinal fusion model
}

\author{
Tetsuo Hayashi, MD, PhD, ${ }^{1,2}$ Elizabeth L. Lord, MD, ${ }^{1}$ Akinobu Suzuki, MD, PhD, ${ }^{1}$ \\ Shinji Takahashi, MD, PhD, ${ }^{1}$ Trevor P. Scott, MD, ${ }^{1}$ Kevin Phan, MD, ${ }^{1}$ Haijun Tian, MD, ${ }^{1}$ \\ Michael D. Daubs, MD, ${ }^{3}$ Keiichiro Shiba, MD, PhD, ${ }^{2}$ and Jeffrey C. Wang, MD ${ }^{4}$ \\ 'Department of Orthopaedic Surgery, University of California, Los Angeles, California; ' 2 Department of Orthopaedic Surgery, \\ Japan Labour Health and Welfare Organization, Spinal Injuries Center, Fukuoka, Japan; ' 2 Department of Orthopaedic Surgery, \\ University of Nevada, Las Vegas, Nevada; and ${ }^{4}$ Department of Orthopaedic Surgery, University of Southern California, Los \\ Angeles, California
}

OBJECTIVE The efficacy of some demineralized bone matrix (DBM) substances has been demonstrated in the spinal fusion of rats; however, no previous comparative study has reported the efficacy of DBM with human mesenchymal stem cells (hMSCs). There is an added cost to the products with stem cells, which should be justified by improved osteogenic potential. The purpose of this study is to prospectively compare the fusion rates of 3 different commercially available DBM substances, both with and without hMSCs.

METHODS Posterolateral fusion was performed in 32 mature athymic nude rats. Three groups of 8 rats were implanted with 1 of 3 DBMs: Trinity Evolution (DBM with stem cells), Grafton (DBM without stem cells), or DBX (DBM without stem cells). A fourth group with no implanted material was used as a control group. Radiographs were obtained at 2, 4, and 8 weeks. The rats were euthanized at 8 weeks. Overall fusion was determined by manual palpation and micro-CT.

RESULTS The fusion rates at 8 weeks on the radiographs for Trinity Evolution, Grafton, and DBX were 8 of 8 rats, 3 of 8 rats, and 5 of 8 rats, respectively. A significant difference was found between Trinity Evolution and Grafton $(p=0.01)$. The overall fusion rates as determined by micro-CT and manual palpation for Trinity Evolution, Grafton, and DBX were 4 of 8 rats, 3 of 8 rats, and 3 of 8 rats, respectively. The Trinity Evolution substance had the highest overall fusion rate, however no significant difference was found between groups.

CONCLUSIONS The efficacies of these DBM substances are demonstrated; however, the advantage of DBM with hMSCs could not be found in terms of posterolateral fusion. When evaluating spinal fusion using DBM substances, CT analysis is necessary in order to not overestimate fusion.

http://thejns.org/doi/abs/10.3171/2015.12.SPINE15737

KEY WORDS demineralized bone matrix; human mesenchymal stem cells; spinal fusion; athymic nude rats; micro-CT; fusion rate; posterolateral fusion

$\mathrm{S}$ PINAL fusion is widely performed in patients with spinal trauma, degenerative disease, and tumors. Autologous iliac crest bone grafting is considered the gold-standard graft choice for spinal arthrodesis; however, it is associated with donor-site morbidity and limited graft supplies. ${ }^{15}$ Bone graft alternatives to replace autograft and augment arthrodesis are a topic of research, as the optimal alternative has yet to be determined. ${ }^{2}$
Several demineralized bone matrix (DBM) products are commercially available for use in spinal fusion surgery. Each product has different osteoinductive proteins due to different methods of preparation, storage, and donor specifications. ${ }^{2,3}$ The efficacy of some DBM substances has been demonstrated in the spinal fusion of rats. ${ }^{8,13,16}$ Previous reports ${ }^{4,12}$ have supported the efficacy of DBM with human mesenchymal stem cells (hMSCs); however, 
no previous comparative study has reported the efficacy of DBM with hMSCs for spinal fusion in vivo. There is an added cost to products with stem cells that should be justified by improved osteogenic potential. We hypothesized that DBM with hMSCs might improve the fusion rate of spinal arthrodesis. The purpose of this study is to prospectively compare the fusion rates of 3 different commercially available DBM substances, both with and without hMSCs, in an athymic rodent spinal fusion model.

\section{Methods}

Thirty-two mature (12-week-old) athymic nude female rats were used in our study. The animals were divided into 4 experimental groups of 8 animals each. Group 1 received Trinity Evolution (DBM with stem cells; Orthofix); Group 2 received Grafton (DBM without stem cells; BioHorizons); Group 3 received DBX (DBM without stem cells; Musculoskeletal Transplant Foundation, available through Synthes); and Group 4 underwent decortication alone without the implantation of any graft material (control group). A posterolateral fusion procedure was performed, and each animal in Groups 1 to 3 was implanted with these commercially available DBM products. These DBMs were obtained in the sterile, factory-sealed packaging for use in humans. Each was obtained directly from the operating room, unopened, and ready for implantation. All products used from 1 company had the same lot number, demonstrating that the material was prepared from the same cadaver bone. All protocols used for this experiment were approved by the animal research committee at our institution.

\section{Product Information}

Trinity Evolution (Group 1) is an allograft that comprises cancellous bone with viable osteogenic and osteoprogenitor cells retained within the matrix and a demineralized cortical bone component. Adult MSCs are multipotential and capable of responding to their environment and differentiating into a variety of cells as needed. ${ }^{11}$ When implanted in patients, MSCs and osteoprogenitor cells do not stimulate an immune response.,11 Trinity Evolution is processed by the Musculoskeletal Transplant Foundation, a nonprofit organization.

Grafton DBM Putty (Group 2) is derived from humanbanked bone tissue. Donors undergo serological and microbiological testing, as well as screening on the basis of their medical and social history. The allograft bone is harvested in a sterile manner, washed, sonicated, treated with antibiotics, and demineralized to contain $<0.5 \%$ calcium phosphate. It is then combined with glycerol, which results in a putty-like consistency. Grafton Putty also undergoes a validated proprietary production process that has been shown to inactivate human immunodeficiency virus-1, hepatitis B virus, hepatitis $\mathrm{C}$ virus, cytomegalovirus, and poliomyelitis virus. ${ }^{1}$ Grafton Putty is packaged in a readyto-use form, and it can be molded or packed directly into a bone defect.

DBX DBM Putty (Group 3) is also derived from human-banked bone tissue. Donors undergo screening procedures as dictated by the Musculoskeletal Transplant
Foundation. Allograft tissue is harvested under sterile conditions, washed, and treated with antibiotics. The tissue is then demineralized with hydrochloric acid so that the resulting bone matrix contains $<8 \%$ calcium. The demineralized bone is combined with a $4 \%$ sodium hyaluronate carrier prior to packaging. DBX is packaged in a syringe, and it can be easily measured and is injected directly into a bone defect site.

\section{Surgical Procedures For Spinal Fusion}

The animals were premedicated with sustained-release buprenorphine 30 minutes prior to surgery and anesthetized with $2 \%$ isoflurane administered in oxygen $(1 \mathrm{~L} /$ minute). This spine fusion model has been previously described. ${ }^{6,8,13,16}$ A midline incision was made in the skin, and the transverse processes of L-4 and L-5 were exposed and bilaterally decorticated with a high-speed bur. After this, $0.3 \mathrm{~cm}^{3}$ of graft material was implanted on each side (total $0.6 \mathrm{~cm}^{3}$ ). The appropriate DBM was implanted into the 8 animals in each of the 3 experimental groups. The control group underwent decortication alone. According to a preset schedule, all the animals were killed at 8 weeks.

\section{Radiographic Analysis}

Posteroanterior radiographs of the lumbar spine were taken of each animal at 2, 4, and 8 weeks after surgery using a Faxitron LX60 cabinet radiography system and evaluated in a blinded fashion by 2 independent observers. Characterization of osseous union was determined by radiographic evidence of bridging, i.e., trabecular bone between the transverse processes. ${ }^{10,16}$

\section{Manual Testing}

Eight weeks after surgery, the animals were killed and their spines were surgically removed. The explanted lumbar spines were manually tested for motion between levels by 2 independent observers who were blinded to the treatments. Any motion detected between the facets or between the transverse processes of L- 4 and L-5 by manual testing was considered a failure of fusion. The absence of motion was considered successful fusion..$^{10,16}$ Manual palpation testing has been reported to the most sensitive and specific method for assessing fusion in this model., ${ }^{9}, 10,14,16$

\section{Micro-CT}

Each excised spine was analyzed by high-resolution micro-CT using a SkyScan 1172 scanner with a voxel isotropic resolution of $20 \mathrm{~mm}$ and an x-ray energy of $55 \mathrm{kVp}$ and $181 \mathrm{~mA}$ in order to further assess the fusion rate and observe the fusion mass as reported. ${ }^{5,10}$ A total of 360 projections were acquired over an angular range of $180^{\circ}$ with steps of $0.5^{\circ}$ and an exposure time of $220 \mathrm{msec} / \mathrm{slice}$. Five frames were averaged at each rotation step in order to get a better signal-to-noise ratio. A $0.5-\mathrm{mm}$ aluminum filter was used to narrow down the X-ray beam frequency and minimize beam-hardening artifacts. Virtual image slices were reconstructed using cone-beam reconstruction software (version 2.6) based on the Feldkamp algorithm (SkyScan). These settings produced serial cross-sectional 1024 $\times 1024-$ pixel images. Sample reorientation and 2D visu- 
TABLE 1. Progression of the fusion rates as determined by radiography at 2,4 , and 8 weeks after surgery

\begin{tabular}{ccccc}
\hline & \multicolumn{4}{c}{ No. of Rats } \\
\cline { 2 - 5 } Time Point & Trinity & Grafton & DBX & Control \\
\hline 2 wks & $5 / 8$ & $0 / 8$ & $0 / 8$ & $0 / 0$ \\
\hline 4 wks & $7 / 8$ & $3 / 8$ & $3 / 8$ & $0 / 0$ \\
\hline 8 wks & $8 / 8$ & $3 / 8$ & $5 / 8$ & $0 / 0$ \\
\hline
\end{tabular}

alization were performed using DataViewer (SkyScan). Fusion was defined as the unilateral presence of bridging bone between the L- 4 and L-5 transverse processes. The reconstructed images were judged to be fused or not fused by 2 experienced independent observers.

\section{Statistical Analysis}

Data from radiography, manual testing, and micro-CT were analyzed using the Fisher exact test since all such comparisons were between 2 independent groups of rats. Statistical analyses were performed using SPSS (version 20 ; SPSS) computer software. A p value $<0.05$ was considered significant for any one comparison.

\section{Results}

The results of the fusion rates, as determined on radiographs at 2, 4, and 8 weeks, are presented in Table 1. Trinity Evolution DBM had a higher fusion rate than the other DBM products at any time point. The fusion rates gradually increased over time in all DBM products. The control group had a $0 \%$ fusion rate.

Fusion rates at 8 weeks, as determined on radiography, manual testing, and micro-CT, are shown in Table 2 . The fusion rates at 8 weeks on radiography for Trinity Evolution, Grafton, and DBX were 8 of 8 rats, 3 of 8 rats, and 5 of 8 rats, respectively. A significant difference was found between Trinity Evolution and Grafton $(p=0.01)$. The fusion rates determined by micro-CT were similar to those by manual palpation testing. The overall fusion rates, as determined by micro-CT and manual palpation testing for Trinity Evolution, Grafton, and DBX were 4 of 8 rats, 3 of 8 rats, and 3 of 8 rats, respectively. The Trinity Evolution substance had the highest overall fusion rate; however, no significant difference was found between groups. In terms of the method of analysis, there is a significant difference between radiographs at 8 weeks ( 8 of 8 rats) and micro-CT (4 of 8 rats) for Trinity Evolution ( $\mathrm{p}=0.03$ ), indicating that we overestimated fusion on radiography (Fig. 1).

TABLE 2. Fusion rates as determined by radiography, micro-CT, and manual palpation testing at 8 weeks

\begin{tabular}{lcccc}
\hline \multirow{2}{*}{ Test } & \multicolumn{4}{c}{ No. of Rats } \\
\cline { 2 - 5 } & Trinity & Grafton & DBX & p Value \\
\hline Radiography & $8 / 8$ & $3 / 8$ & $5 / 8$ & $0.01^{*}$ \\
\hline Manual testing & $4 / 8$ & $3 / 8$ & $3 / 8$ & NS \\
\hline Micro-CT & $4 / 8$ & $3 / 8$ & $3 / 8$ & NS \\
\hline
\end{tabular}

NS = not significant.

* A significant difference was found between Trinity and Grafton.
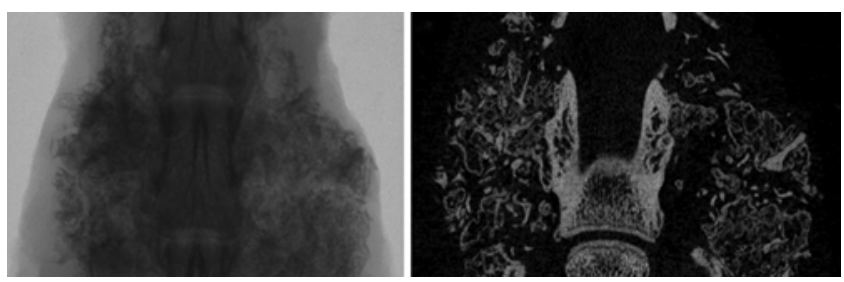

FIG. 1. The radiograph (left) was judged as fusion; however, micro-CT (right) revealed the nonfusion of Trinity Evolution.

\section{Discussion}

Human MSC with biomaterials was reported to have a certain potential for spinal fusion. Barbanti Brodano et al. ${ }^{4}$ demonstrated the benefits of hMSCs and biomaterial synergy for facilitating spinal fusion in vitro. The efficacy of hMSCs in vivo, however, has not been thoroughly demonstrated. We demonstrated that DBM with hMSCs had a higher rate of spinal fusion than other DBM products without hMSCs; however, no significant difference was found between the groups by either manual testing or micro-CT.

Several previous reports have explored the fusion rates of commercially available DBM without hMSCs. Peterson et al. ${ }^{13}$ compared 3 commercially available DBM products and found that Grafton Putty resulted in a higher fusion rate than DBX putty, and that Allomatrix Injectable Putty (Wright Medical Technology) failed to promote fusion. Similarly, Wang et al. ${ }^{16}$ compared the osteoinductive potential of 3 DBM products (Osteofil [Regeneration Technologies], Grafton, and Dynagraft [GenSci OrthoBiologics]) and rodent autologous iliac crest bone graft in an athymic rat model of posterolateral fusion and found that Dynagraft did not produce fusion at any time point. Lee et al. ${ }^{8}$ compared 8 different commercially available DBM products in athymic rats and found that Osteofil Paste and Grafton putty demonstrated the highest fusion rates, and Allomatrix demonstrated the lowest. Our results were similar to previous reports; however, commercially available DBM products exhibit significant variability in fusion performance that is secondary to differences in carrier media and processing.

Several limitations to this study must be acknowledged. Variability in DBM products has been reported in terms of both BMP concentrations and the in vivo rates of fusion. ${ }^{3}$ This variability may affect the comparison of fusion rates among those DBM products. In addition, although we could not find any significance in terms of the fusion rate in this sample size, a larger number of rats in each group should be further evaluated to increase the power of this study. Also, although we could not find any significant difference because of the respective DBM substrates that were tested, it is difficult to assess the contribution of hMSCs alone to fusion. Human cells were implanted into the rats in this study; however, hMSCs may not differentiate in a rat model, even after accounting for athymic rats. Xenotransplantation may affect the bioactivity of hMSCs.

The reason why Trinity Evolution seemed to result in a higher rate of fusion on radiography appears to be its radiopacity. The radiographic progression of the groups is shown in Fig. 2. Unlike Grafton or DBX, the radiographs 
T. Hayashi et al.
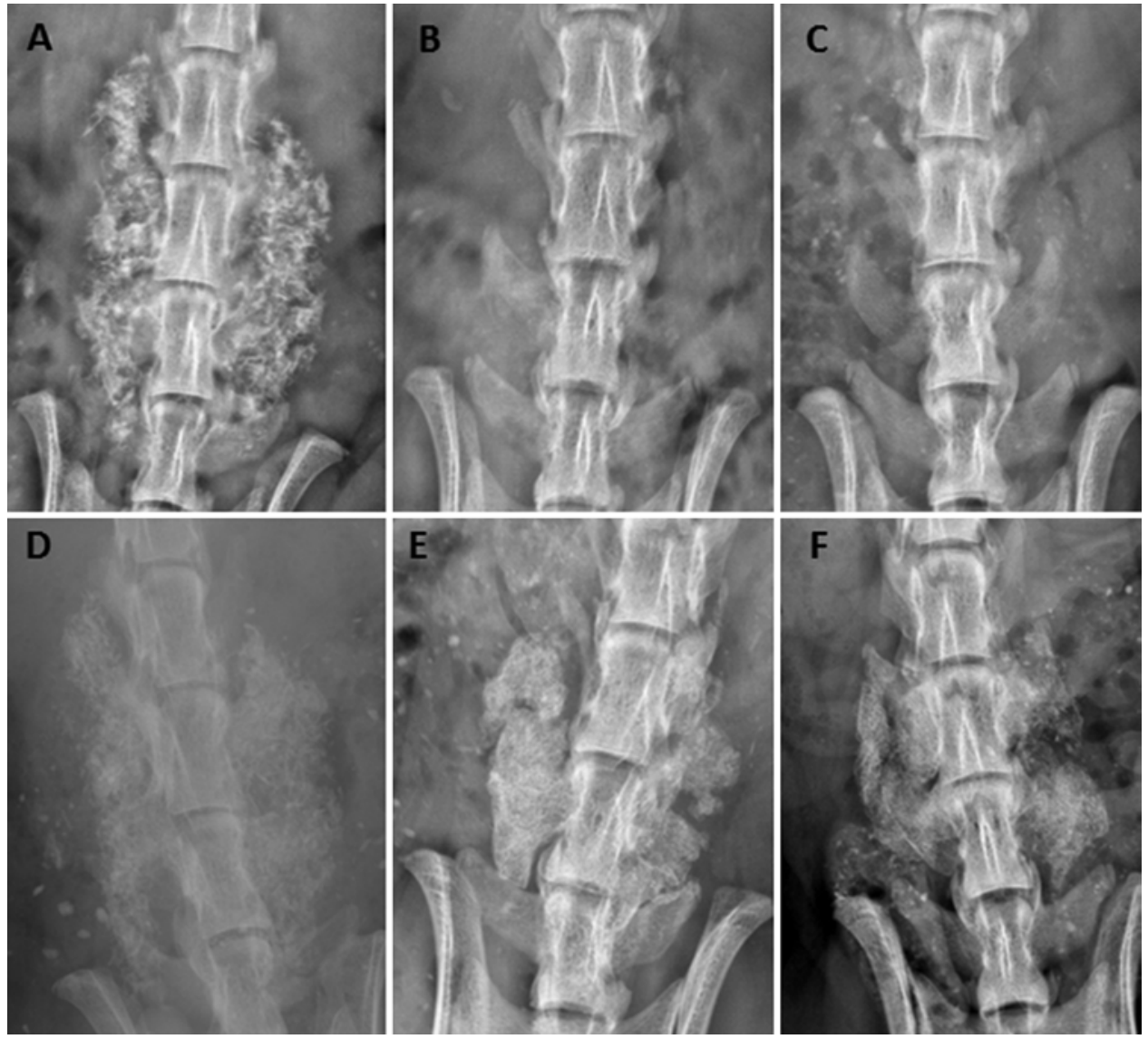

FIG. 2. Radiographs of Trinity, Grafton, and DBX at 2 weeks after surgery (A, B, and C, respectively) and 8 weeks after surgery $(D, E$, and $F$, respectively). The radiographs of the Trinity Evolution Group at even 2 weeks after surgery (A) showed a fusion-like appearance.

for Trinity Evolution at postoperative Week 2 showed a fusion-like appearance. At this time point, it is unlikely that this was indicative of actual fusion; more likely, this composition of the material is opaque enough on radiography to be misjudged as fusion. Thus, detailed evaluation using CT scanning is necessary to judge actual fusion.

\section{Conclusions}

We demonstrated the efficacy of 3 DBM substances; however, there was no advantage of adding hMSCs, which is more expensive. When we evaluate spinal fusion using DBM substance, CT analysis is necessary in order to not overestimate fusion.

\section{References}

1. Ad Hoc Working Party on Biotechnology/Pharmacy: Validation of virus removal and inactivation procedures. Pharmacol Toxicol 69:144-148, 1991

2. Aghdasi B, Montgomery SR, Daubs MD, Wang JC: A review of demineralized bone matrices for spinal fusion: the evidence for efficacy. Surgeon 11:39-48, 2013

3. Bae H, Zhao L, Zhu D, Kanim LE, Wang JC, Delamarter RB: Variability across ten production lots of a single demineralized bone matrix product. J Bone Joint Surg Am 92:427-435, 2010
4. Barbanti Brodano G, Mazzoni E, Tognon M, Griffoni C, Manfrini M: Human mesenchymal stem cells and biomaterials interaction: a promising synergy to improve spine fusion. Eur Spine J 21 (Suppl 1):S3-S9, 2012

5. Johnson JS, Meliton V, Kim WK, Lee KB, Wang JC, Nguyen $\mathrm{K}$, et al: Novel oxysterols have pro-osteogenic and antiadipogenic effects in vitro and induce spinal fusion in vivo. $\mathbf{J}$ Cell Biochem 112:1673-1684, 2011

6. Kim HS, Viggeswarapu M, Boden SD, Liu Y, Hair GA, Louis-Ugbo J, et al: Overcoming the immune response to permit ex vivo gene therapy for spine fusion with human type 5 adenoviral delivery of the LIM mineralization protein-1 cDNA. Spine (Phila Pa 1976) 28:219-226, 2003

7. Le Blanc K, Tammik C, Rosendahl K, Zetterberg E, Ringdén O: HLA expression and immunologic properties of differentiated and undifferentiated mesenchymal stem cells. Exp Hematol 31:890-896, 2003

8. Lee YP, Jo M, Luna M, Chien B, Lieberman JR, Wang JC: The efficacy of different commercially available demineralized bone matrix substances in an athymic rat model. J Spinal Disord Tech 18:439-444, 2005

9. Miyazaki M, Morishita Y, He W, Hu M, Sintuu C, Hymanson $\mathrm{HJ}$, et al: A porcine collagen-derived matrix as a carrier for recombinant human bone morphogenetic protein-2 enhances spinal fusion in rats. Spine J 9:22-30, 2009

10. Montgomery SR, Nargizyan T, Meliton V, Nachtergaele S, Rohatgi R, Stappenbeck F, et al: A novel osteogenic oxysterol compound for therapeutic development to promote bone growth: activation of hedgehog signaling and osteogenesis 
through smoothened binding. J Bone Miner Res 29:18721885,2014

11. Niemeyer P, Krause U, Kasten P, Kreuz PC, Henle P, Südkam NP, et al: Mesenchymal stem cell-based HLA-independent cell therapy for tissue engineering of bone and cartilage.

Curr Stem Cell Res Ther 1:21-27, 2006

12. Park BW, Kang EJ, Byun JH, Son MG, Kim HJ, Hah YS, et al: In vitro and in vivo osteogenesis of human mesenchymal stem cells derived from skin, bone marrow and dental follicle tissues. Differentiation 83:249-259, 2012

13. Peterson B, Whang PG, Iglesias R, Wang JC, Lieberman JR: Osteoinductivity of commercially available demineralized bone matrix. Preparations in a spine fusion model. J Bone Joint Surg Am 86-A:2243-2250, 2004

14. Schimandle JH, Boden SD: Spine update. The use of animal models to study spinal fusion. Spine (Phila Pa 1976) 19:1998-2006, 1994

15. Silber JS, Anderson DG, Daffner SD, Brislin BT, Leland JM, Hilibrand AS, et al: Donor site morbidity after anterior iliac crest bone harvest for single-level anterior cervical discectomy and fusion. Spine (Phila Pa 1976) 28:134-139, 2003

16. Wang JC, Alanay A, Mark D, Kanim LE, Campbell PA, Dawson EG, et al: A comparison of commercially available demineralized bone matrix for spinal fusion. Eur Spine J 16:1233-1240, 2007

\section{Disclosures}

Dr. Daubs holds a patent with DePuy Synthes.

\section{Author Contributions}

Conception and design: Hayashi, Suzuki, Scott, Phan, Wang. Acquisition of data: Hayashi, Takahashi, Scott, Phan. Analysis and interpretation of data: Hayashi, Takahashi. Drafting the article: Hayashi, Lord, Scott. Critically revising the article: Hayashi, Lord, Suzuki, Scott, Tian. Reviewed submitted version of manuscript: Hayashi, Lord, Suzuki, Takahashi. Approved the final version of the manuscript on behalf of all authors: Hayashi. Statistical analysis: Hayashi, Takahashi. Administrative/techni$\mathrm{cal} /$ material support: Lord, Suzuki, Takahashi, Scott, Tian, Wang. Study supervision: Suzuki, Daubs, Shiba, Wang.

\section{Correspondence}

Tetsuo Hayashi, Department of Orthopaedic Surgery, Japan Labour Health and Welfare Organization, Spinal Injuries Center, 550-4 Igisu, Iizuka city, Fukuoka 820-8508, Japan. email: tetsuo884hayashi@yahoo.co.jp. 